\title{
Effectiveness and Safety of Fentanyl Compared with Morphine for Out-of-Hospital Analgesia
}

\author{
Ross J. Fleischman, MD, David G. Frazer, BS, Mohamud Daya, MD, MS, Jonathan Jui, MD, \\ MPH, and Craig D. Newgard, MD, MPH \\ Center for Policy and Research in Emergency Medicine, Department of Emergency Medicine, \\ Oregon Health \& Science University, Portland, Oregon
}

\begin{abstract}
Background-Fentanyl has several potential advantages for out-of-hospital analgesia, including rapid onset, short duration, and less histamine release.

Objective-To compare the effectiveness and safety of fentanyl with that of morphine.

Methods-This was a retrospective before-and-after study of a protocol change from morphine to fentanyl in an advanced life support emergency medical services system in January 2007. Charts from nine months prior to the change and for nine months afterward were abstracted by two reviewers using a standardized instrument. The first three months after the change were excluded. Effectiveness was measured by change in pain scores on a $0-10$ scale. A priori-defined adverse events included out-of-hospital events: respiratory rate $<12$ breaths/min, pulse oximetry $<92 \%$, systolic blood pressure $<90 \mathrm{mmHg}$, any fall in Glasgow Coma Scale score, nausea or vomiting, intubation, and use of antiemetic agents or naloxone. Emergency department charts were reviewed for initial pain scores and the same adverse events during the first two hours. Events clearly not attributable to the opioid were discounted. The changes in pain scores were also compared adjusting for confounders by multivariable linear regression.
\end{abstract}

Results-Three hundred fifty-five patients aged 13 to 99 years received morphine during the nine months before the protocol change and 363 received fentanyl following the washout period. Initial pain scores for morphine (8.1) and fentanyl (8.3) were comparable (95\% confidence interval [CI] for difference -1.1 to 0.3 ). Fentanyl patients received a higher equivalent dose of opioid (7.7 mg morphine equivalents for morphine, $9.2 \mathrm{mg}$ for fentanyl, CI for the difference 0.9 to 2.3). The mean decreases in pain score were similar between the drugs (2.9 for morphine, 3.1 for fentanyl, CI for the difference -0.3 to 0.7 ). With regard to adverse events, $9.9 \%$ of the morphine patients and $6.6 \%$ of the fentanyl patients experienced an adverse event in the field (CI for the difference -0.8 to $7.3 \%$ ). The most common event was nausea, with a rate of $7.0 \%$ for morphine vs. $3.8 \%$ for fentanyl (CI for the difference $-0.1 \%$ to $6.5 \%$ ).

Conclusion-Morphine and fentanyl provide similar degrees of out-of-hospital analgesia, although this was achieved with a higher dose of fentanyl. Both medications had low rates of adverse events, which were easily controlled.

\footnotetext{
Address correspondence and reprint requests to: Ross J. Fleischman, MD, Department of Emergency Medicine, Oregon Health \& Science University, 3181 SW Sam Jackson Park Road, Mail Code CR114, Portland, OR 97239. fleischr@ohsu.edu. Presented as an abstract at the Society for Academic Emergency Medicine annual meeting, New Orleans, Louisiana, May 2009.

This article is not subject to U.S. copyright law.

The authors report no conflicts of interest. The authors alone are responsible for the content and writing of this paper.
} 


\section{Keywords}

analgesia; fentanyl; morphine; analgesics; opioid; prehospital emergency care; emergency medical services

\section{Introduction}

Pain control is widely recognized as a goal of emergency medical care. The inadequacy of pain control in the emergency department (ED) has been the subject of much research. ${ }^{1}$ To a lesser extent, this attention has also been focused on analgesia in the out- of-hospital setting. In 2003, the National Association of EMS Physicians issued a position statement recommending protocols for pain management including formal assessment of pain and administration of analgesic pain medication. ${ }^{2}$

Considerable variability exists in out-of-hospital pain management protocols nationwide. While a recent survey of state emergency medical services (EMS) directors found that $78 \%$ allowed opioids without contact with a physician, the type of opioid used in the out-ofhospital setting varied. ${ }^{3}$ In the same survey, $48 \%$ allowed the use of fentanyl.

Whereas morphine has a proven record of safety and effectiveness in the out-of-hospital setting, fentanyl has many characteristics that theoretically make it even more desirable. Among these are its short time to peak effect, short duration of action, and minimal provocation of histamine release, which causes itching and hypotension. Fentanyl has also been touted as causing less nausea compared with morphine, although this has not been rigorously validated. ${ }^{4,5}$ The preferred agent to control pain in the out-of-hospital setting, while also minimizing adverse events, remains unclear.

We sought to examine the comparative effectiveness (pain control and adverse events) of morphine versus fentanyl for out-of-hospital analgesia in a before -and-after study based on a planned EMS protocol change from morphine to fentanyl.

\section{Methods}

\section{Study Design}

This was a retrospective before-and-after study of a countywide EMS protocol change from morphine to fentanyl. Multnomah County, Oregon, with a population of approximately 700,000 and an area of 466 square miles, is served by multiple first-responding advanced life support fire agencies, with a single commercial advanced life support ambulance company transporting patients.

\section{Study Population and Setting}

The study population included a representative sample of patients aged 13 years and above receiving intravenous (IV) morphine from March 1 through December 31, 2006, or IV fentanyl from March 1 through December 31, 2007, for pain. Throughout 2006, morphine, which had been used for several years, was the only opioid analgesic available to out-ofhospital providers. On January 1, 2007, morphine was replaced systemwide by fentanyl. The first three months after fentanyl was introduced in 2007 were excluded as a washout period to decrease the biases associated with using a new medication.

Children under the age of 13 years were excluded because 13 years is the age down to which the feasibility of using an out-of-hospital numeric rating scale has been demonstrated. ${ }^{6}$ Only patients being transported from the scene of injury or illness to one of four destination 
hospitals were included. These hospitals were selected to provide a range of facility types, including an academic medical center that is one of the state's two level I trauma centers, two large community hospitals, and a smaller suburban community hospital. These four hospitals received $40 \%$ of the patients aged 13 years and older receiving morphine or fentanyl during the study period. Interhospital transfers were excluded since many such patients had already received analgesics prior to transfer.

\section{Human Subjects Review}

The study was granted a Health Insurance Portability and Accountability Act (HIPAA) waiver of informed consent by the Oregon Health \& Science University and Providence Health System institutional review boards.

\section{Study Protocol}

The existing EMS analgesic protocol allowed medication administration (morphine or fentanyl) by paramedics without direct oversight for isolated extremity injuries, burns, and suspected ischemic chest pain unresponsive to nitroglycerin. Morphine was given (in 2006) as an IV dose of $2--5 \mathrm{mg}$, repeated every 5 minutes to a maximum of $20 \mathrm{mg}$. Fentanyl was given (in 2007) as a 50- $\mu \mathrm{g}$ IV dose, with repeated doses of 25--50 $\mu \mathrm{g} 3--5$ minutes to a maximum of $200 \mu \mathrm{g}$. This change in protocol allowed paramedic discretion to administer morphine at an initial dose range that was therapeutically smaller than the fixed initial dose for fentanyl based on an equivalence of $5 \mathrm{mg}$ morphine to $50 \mu \mathrm{g}$ fentanyl. ${ }^{7}$ Pediatric patients 13 years and older received morphine at $0.1 \mathrm{mg} / \mathrm{kg}$ or fentanyl at $1 \mu \mathrm{g} / \mathrm{kg}$ body weight. Online medical control (OLMC) was required for additional dosing once the maximum amount had been reached.

Eligible cases were identified by searching the ambulance service's patient care record database for all cases of morphine or fentanyl administration during the time periods under review. All EMS documentation was performed electronically throughout the county using a proprietary electronic patient care charting system. All data were abstracted from EMS and ED patient care reports using a standardized data form in Microsoft Access 2007 (Microsoft Corporation, Redmond, WA) that was pilot tested and refined for this project by the investigators.

\section{Measurements}

Effectiveness was measured by the change in pain scores on a $0-10$ verbal numeric rating scale from the first (prior to medication) to the last measurement by EMS following analgesia administration. Pain scores documented as decimals were rounded down to the nearest whole number. Pain scores documented as greater than 10 were abstracted as 10 . Reports of "no pain" were scored as 0 . Adverse events included systolic blood pressure under $90 \mathrm{mmHg}$, respiratory rate under 12 breaths/min, oxygen saturation $\left(\mathrm{SpO}_{2}\right)$ below $92 \%$ and $5 \%$ below baseline, and any decrease in the Glasgow Coma Scale (GCS) score following administration of the opioid. We defined hypoxemia as an $\mathrm{SpO}_{2}<92 \%$, as a point at which a provider would likely intervene to prevent further oxygen desaturation. This value falls between the physiologic definition of hypoxemia corresponding to an $\mathrm{SpO}_{2}$ of $90 \%$ and the definition of hypoxemia in the ED sedation literature as below $93 \% .{ }^{8,9} \mathrm{We}$ arbitrarily chose a decrease of $5 \%$ below baseline in order that patients who were nearly hypoxemic at baseline (e.g., with an $\mathrm{SpO}_{2}$ of 93\%) and then had an insignificant desaturation (e.g., to $91 \%$ ) would not be misclassified as having an adverse event attributable to the opioid. Nausea and vomiting were assessed by the notation of these findings or the administration of antiemetic medications following opioid therapy. Charts were also reviewed for the need for interventions suggestive of oversedation, including 
ventilatory support (e.g., bag-valve-mask ventilation), intubation, or the administration of naloxone.

All charts were initially reviewed by one of the first two authors. The $20 \%$ of charts found to have a possible adverse event or possible inconsistency in the data on initial review were marked for a second review. These charts were then reexamined by both reviewers to check for accurate transcription and to exclude adverse events that were discounted as clearly not attributable to the opioid medication according to three explicit criteria defined during the pilot testing of the instrument: 1) For the vital sign criteria, we considered any abnormal vital sign that was present prior to administration of the opioid not to be attributable to the opioid. 2) Documented complaints such as nausea and vomiting were not considered to be adverse events if the written history of the present illness clearly documented them to be present prior to the administration of the opioid. 3) Antiemetic agents that were clearly documented in the free-text narrative as being given for prophylactic purposes without preceding nausea were not considered adverse events. All potential adverse events not clearly meeting these three explicit criteria were considered to be adverse events.

Out-of-hospital charts were also abstracted for other patient characteristics and details of the EMS run. Chief complaints were grouped as extremity/hip pain and burns, suspected ischemic chest pain, other chest pain, atraumatic abdominal and pelvic pain, back pain, and other head and neck pain. Mechanisms of injury were categorized as a fall from ground level or down stairs, fall from height, lifting/straining/reaching or repetitive motion injury, motor vehicle crash, motorcycle or all-terrain vehicle crash, bicycle and other nonmotorized vehicle crash, or chronic pain. The destination hospital was recorded, as was whether the opioid was given for an on-protocol indication or required OLMC approval. Past medical histories were abstracted from the out-of-hospital chart in the categories used for Deyo's calculation of the Charlson co-morbidity score, a validated prognostic instrument for survival based on 17 weighted medical diagnoses. ${ }^{10}$ Medications were classified into one of 41 predetermined categories within the Anatomical Therapeutic Chemical classification, with the most specificity given to relevant medication such as home opioids and nonopioid analgesics. ${ }^{11}$ All out-of-hospital vital signs were recorded including blood pressure, respiratory rate, pulse rate, and $\mathrm{SpO}_{2}$. Patient weight was by paramedic estimate. The dose and timing of all medications administered out of hospital were recorded.

We reviewed the ED notes for the first pain score (if documented using the standard 0--10 numeric rating scale within the first hour after arrival) as well as for the same markers of adverse events occurring within the first two hours of the visit.

\section{Analytical Methods}

Our sample size calculation for the effectiveness of morphine compared with fentanyl was based on a difference of 1.4 points (effect size) on a numeric rating scale, falling between the minimum clinically significant differences of 1.3 and 1.5 reported in two ED pain studies. ${ }^{12,13}$ This difference was similar to the $14-\mathrm{mm}$ minimum significant difference on a $100-\mathrm{mm}$ visual analog scale used by Galinski et al. in a randomized trial of morphine versus fentanyl for out-of-hospital analgesia. ${ }^{14}$ While the numeric and visual rating scales are not equivalent, ED studies have shown a close correlation between the two. ${ }^{15}$ Using a $5 \%$ level of significance $(\alpha), 80 \%$ power $(\beta=0.20)$, and planned two- sided comparison of means, we calculated a minimum requirement of 45 patients per arm. We far exceeded this sample size in order that our analysis for adverse events would be as robust as possible. The time periods (nine months for each) selected for query of EMS records were based on systematic identification of patients treated by EMS during each analgesia protocol. 
Prior studies have shown combined rates between $0 \%$ and $2 \%$ of hemodynamic, respiratory, or level-of- consciousness effects. ${ }^{12,16--19}$ Given the extraordinarily large sample sizes that would be necessary to demonstrate a difference between such low rates, we did not attempt to power our study to show an adverse event difference that would not have been clinically significant; rather, we felt that demonstrating that the rates of serious adverse events were very low was sufficient to support the safety of either drug. Adverse events were secondary outcomes in this study.

Differences for categorical variables between the two medication groups were calculated by the chi-square test using a p-value of 0.05 ; continuous variables were analyzed using the two-sample t-test for means.

Multivariable linear regression was also used to assess the change in pain scores between medication groups, after adjusting for confounders. Confounders for the multivariable model were selected based on factors we expected to be predictors of pain scores as well as factors we found to be significantly different between the treatment groups in univariate testing. We used $\alpha \leq 0.05$ as the level of significance.

To reduce bias and preserve study power, multiple imputation was used for missing out-ofhospital and/or ED pain scores. No other study variables were imputed. Multiple imputation was performed using the sequential regression imputation method using IVEware (May 2009, Survey Methodology Program, University of Michigan) ${ }^{20}$ This technique generates a range of plausible values for each missing value, based on correlations within the data. All data present were used as predictors for the missing values. The use of multiple imputation has been shown to reduce bias and preserve study power and has been validated for out-ofhospital studies. ${ }^{21} \_23$

Data cleaning and analyses were performed using SAS (SAS version 9.1, SAS Institute, Inc., Cary, NC).

\section{Results}

The study population consisted of 718 patients aged 13--99 years, 355 of whom received morphine and 363 fentanyl. The morphine- and fentanyl-treated groups were not significantly different in gender, age, Charl- son comorbidity score, mechanism (for injured patients), baseline vital signs, or baseline pain scores (Table 1). Significant differences were seen in chief complaint, destination hospital, on-protocol versus OLMC-approved indications, and home use of certain oral opioids. Fentanyl patients received a higher narcotic equivalent dose of opioid (7.7 mg morphine equivalents for morphine, $9.2 \mathrm{mg}$ for fentanyl; confidence interval [CI] for difference 0.9 to 2.3) but the same number of doses. Narcotic equivalent dosing for fentanyl was also significantly higher per kilogram of estimated body weight.

The mean initial pain scores were 8.1 (CI 7.9 to 8.4) for morphine and 8.3 (CI 8.1 to 8.5) for fentanyl (Fig. 1). The mean decrease of 2.9 (CI 2.5 to 3.2) points on a 10-point numeric rating scale from the first to the last field measurements for morphine patients was not significantly different from the 3.1-point decrease seen for fentanyl cases (CI 2.8 to 3.4). Patients receiving morphine experienced an increase in their pain scores of 0.9 (CI 0.5 to 1.2), which was not significantly different from the increase for fentanyl of 0.8 (CI 0.4 to $1.1)$.

We applied a multivariable linear regression model to adjust for possible confounders. These included predictors that we expected a priori to be significant in predicting the change in pain scores (age, gender, Charlson comorbidity score, initial pain score, log of patient 
weight, and occurrence of adverse events described above), as well as predictors that were significantly different between the two groups (destination hospital, OLMC contact, analgesic equivalent dose, and chief complaint). In this model, there was no significant difference in pain reduction between the two medications (from the first to the last field pain scores, decrease of 0.23 points less for morphine, $95 \% \mathrm{CI}-0.24$ to 0.71 , Table 2). There was also no difference in the change from the last field measurement to the initial ED pain score ( 0.02 points for morphine, $95 \% \mathrm{CI}-0.52$ to 0.56 ).

Both morphine and fentanyl showed low rates of adverse events, with nausea being the most common side effect (Fig. 2). Fifty-four patients had potential side effects that were discounted as not attributable to the opioid medication according to our predefined criteria, 49 of which were cases in which the patient was nauseated prior to receiving the opioid. Ten patients in total had out-of-hospital systolic blood pressures under $90 \mathrm{mmHg}$ that could reasonably be attributed to the opioid therapy. This hypotension resolved spontaneously or with a fluid bolus in all 10 cases. Ten patients became hypoxic or had a respiratory rate below 12 breaths/min, but none required treatment besides supplemental oxygen. Only one patient required naloxone out of hospital: A 54-year-old woman who received $25 \mu \mathrm{g}$ of fentanyl for atraumatic abdominal pain received $1.2 \mathrm{mg}$ of naloxone for a fall in GCS score from 15 to 7 without respiratory depression. No patient in this study required intubation.

Additional adverse events were captured by following patients through the first two hours of their ED stay (Fig. 3). Surveillance into the ED identified adverse events in 51 patients (7.1\%) who had no field-identified adverse events. Two patients received nalox- one in the ED: One patient who received two 50- $\times \mathrm{g}$ doses of fentanyl in the field received naloxone for oxygen saturations falling into the 70s (\%) after an additional 1-mg IV dose of hydromorphone was given in the ED. Another patient who had received $8 \mathrm{mg}$ of morphine in the field required naloxone for apnea after receiving $4 \mathrm{mg}$ IV of hydromorphone, $1 \mathrm{mg}$ IV of lo- razepam, and $2 \mathrm{mg}$ IV of midazolam in the ED. Except for these two patients, none required ED interventions for hypoxia, sedation, or low respiratory rate besides stimulation and supplemental oxygen. No patients required intubation. Six patients had hypotension (systolic blood pressure [SBP] $<90 \mathrm{mmHg}$ ) in the $\mathrm{ED}$, four patients (two receiving morphine and two receiving fentanyl) improved spontaneously to a SBP over $90 \mathrm{mmHg}$, and one morphine patient did so after receiving 1 liter of IV normal saline. The final patient was an 84-year-old woman in hospice for end-stage congestive heart failure who received $5 \mathrm{mg}$ IV of morphine for a ground-level fall resulting in pubic rami fractures. She developed hypotension with a blood pressure as low as $50 / 35 \mathrm{mmHg}$, remained hypotensive through two days of hospitalization for pain control, and died in the hospital. The proportion of patients experiencing an adverse event at any time during either their out-of- hospital or ED phases also showed no significant difference (23.1\% for morphine, $23.8 \%$ for fentanyl; CI for the difference $-6.9 \%$ to $5.5 \%$ ).

As a sensitivity analysis, a complete case analysis was performed excluding cases that did not have two documented numeric pain scores. No missing values were imputed. Results were not qualitatively different (data not shown).

\section{Discussion}

In this study, we demonstrate that morphine and fen- tanyl provide similar degrees of analgesia with similarly low rates of side effects when assessed before and after a countywide EMS protocol change for field analgesia. This analgesia was achieved with higher equivalent doses of fentanyl. Surveillance into the initial ED experience revealed additional adverse events, most of which were related to nausea, though there was still a relatively low overall rate of potentially serious events for either medication group. 
Our results are consistent with those of previous uncontrolled studies demonstrating fentanyl's safety. Kanowitz et al. performed a retrospective analysis of over 2,000 patients who had been given fentanyl for analgesia by out-of-hospital personnel. ${ }^{16}$ They reported a rate of post-fentanyl vital sign abnormalities of only $0.6 \%$, with no deaths or hospitalizations. While this study lacked a control group, it did show a statistically significant reduction in pain score after fentanyl administration, reducing the reported pain level from severe to mild. A study by Nielsen et al. of fentanyl administered by Danish advanced emergency medical technicians showed an overall adverse event rate of 5\%, of which only two cases $(0.4 \%)$ were serious and only one required naloxone. ${ }^{17}$ Again, in the absence of a control group, pain reduction was significant, with an average decrease of five points on a 10-point numeric rating scale. Thomas et al. recorded only four episodes of hypotension, defined as a systolic blood pressure under $90 \mathrm{mmHg}$, following 213 doses of fentanyl given for traumatic pain in the aeromed-ical setting. ${ }^{18}$ Many of the patients in this study had suffered severe, multisystem traumas, which might have been an alternate explanation for the hypotension. In addition, for the 124 doses given to 67 non-intubated patients, none of the patients exhibited clinically significant respiratory depression. In DeVellis et al.'s retrospective review of pediatric cases from the same aeromedical transport service, no hypotension was seen following the 211 doses given to 113 patients; furthermore, no oxygen desaturation was seen in the 52 non-intubated children. ${ }^{19}$

While these studies support the safety of fentanyl for use in EMS, previous research demonstrating its analgesic effectiveness compared with other medications (e.g., morphine) or comparing adverse event rates between analgesics is sparse. Galinski et al. randomized 54 patients to receive repeated doses of morphine or fentanyl as needed for pain control. ${ }^{14}$ They found no significant difference in the efficacy of pain control based on a visual analog rating scale through 30 minutes of follow-up. Congruous with the other studies, they observed no serious side effects and no significant difference in the rate of side effects between the two groups. Similarly, an earlier study by Silv-fast and Saarnivaara randomized 36 EMS patients with ischemic-type chest pain to receive morphine or alfen- tanyl, a congener of fentanyl, with similarly rapid onset but lower potency and shorter duration of action than fentanyl. ${ }^{24}$ Pain control was significantly faster in the group receiving alfentanyl and was significantly better at the final 15-minute time point. No serious side effects or vital sign abnormalities were observed. Weldon et al. demonstrated similar results in a randomized trial of 187 patients who received morphine or fentanyl for ischemic-type chest pain. There were no significant differences between the groups in pain score, mean arterial pressure, heart rate, or respiratory rate. ${ }^{25}$

Our findings expand upon previous out-of-hospital pain research in several ways. First, our before-and- after study design allowed an effectiveness comparison between the two drugs in a paramedic-based EMS setting with a much larger sample size than any of the randomized controlled trials in this area. Second, the Galinski and Silvfast trials were conducted under a European EMS model in which a physician is often present at the prehospital scene, which may not be analogous to EMS systems in North America. Third, the follow-up periods in the Galinski study (30 minutes) and Silvfast study (15 minutes) might not have captured the full effect or all adverse events associated with the drugs. Kanowitz et al. did follow a subset of their patients into the ED setting. Following patients into the ED allowed us to test the hypothesis that fentanyl's duration of action might be so short that its effect could wane before care could be transi-tioned to the ED team and additional narcotics could be given. Our results showed that there was no significant difference in the increase in pain scores from the field to the $\mathrm{ED}(0.83$ for morphine, 0.77 for fen- tanyl). Our results suggest that fentanyl's expected duration of action of 30--60 minutes was adequate to provide sustained pain control during the transition of care ${ }^{26}$ 
Our results show that both morphine and fentanyl can be given by out-of-hospital providers with similar levels of pain relief and a relatively low rate of adverse events. We found that while both drugs were commonly associated with nausea, the rates of more serious adverse events (i.e., hypoxia, hypotension, respiratory depression, and oversedation) were very low. When more serious adverse events did occur, most resolved spontaneously or with simple interventions such as administration of IV fluid or supplemental oxygen. Only three patients required naloxone for reversal of their opioids, two of whom required it after receiving additional sedating medications in the ED. No patient required intubation.

While some differences in adverse event rates did approach statistical significance, this should not be misinterpreted as suggesting the safety of one drug over the other. When adverse event rates were counted across both the out-of-hospital and ED phases, morphine's and fentanyl's safety levels were extremely close, erasing even the suggestion of a trend toward safety in one drug or the other. Our data do not suggest that further work should be done to try to determine which drug is safer: The low absolute rates and small difference between the two drugs make it unlikely that this difference would become either clinically or statistically significant, even with a larger sample size.

Given that the costs of the two drugs are similar, with an average wholesale price of $\$ 0.71$ for $10 \mathrm{mg}$ of morphine versus $\$ 0.83$ for $100 \mu \mathrm{g}$ of fentanyl, our results show no compelling argument for the use of one drug over the other. ${ }^{27}$

\section{Limitations}

A before-and-after study has limitations inherent to a retrospective study design, including the potential to be confounded by differences between the periods; however, our analysis showed the patient groups to be quite similar. Use of our multivariable model further attempted to adjust for the small differences between groups.

We chose a retrospective design in order to eliminate the observer bias associated with medics' knowing they were recording observations for the purpose of a study. This, however, might have resulted in underreporting of adverse events. An additional outcome that our study did not test was itching, which might be less severe with fentanyl because of its decreased his-tamine release and could have been assessed using a prospective instrument.

A second limitation was missing data. Initial numeric pain assessments were missing in $26 \%$ of cases and a subsequent assessment was missing in 33\%. Our rate of missing data was similar to that of another out-of-hospital pain study following introduction of a pain management protocol, which found that $25 \%$ of patients lacked either a documented numeric pain score or any pain assessment at all. ${ }^{6}$ Given that the absence of pain scores does not occur at random, but is confounded by patient characteristics, simply excluding patients with missing pain scores and performing a complete case analysis would have biased study results. ${ }^{28}$ Therefore, we used multiple imputation to preserve sample size and avoid this bias. While this approach attempts to generate missing values and confidence estimates based on all data present, there is still the possibility that the pattern of missing data is not fully explained by the predictors present.

Even though our analysis showed the two patient groups to be similar, our results still could have been confounded by differences in medic behavior. Institution of the new drug (fentanyl) could have made medics more or less aggressive in their dosing or in their reporting of pain scores or adverse effects. Excluding the first three months of fentanyl as a washout period may have mitigated but not eliminated this bias. One potentially important observed difference was that while the two groups received similar numbers of doses, the 
fentanyl group received a greater narcotic equivalent total dose. It is possible that had the doses been truly equivalent, morphine would have been more effective, with a higher rate of adverse events; however, our multiple regression model including narcotic equivalent dose as a potential predictor of the change in pain scores showed a similar result to the unadjusted scores.

Variability in how the verbal numeric rating scale was presented by the paramedics may also have lowered the study's reliability, although it more accurately reflects the real-world effectiveness of these drugs, which was our study's desired outcome. While the numeric rating scale has been validated for research, it has been studied less than the visual analog scale. $^{12}$

Our adverse event rates may have been confounded by therapies given by the paramedics to prevent adverse events, particularly the administration of supplemental oxygen and antiemetic agents. As supplemental oxygen was given at paramedic discretion, earlier administration of oxygen could have confounded which patients crossed the threshold to be considered hypoxic. Protocols also allowed for administration of antiemetic medications for prophylaxis of nausea, which was not considered to be an adverse event if it was clearly documented as such. Differences in prophylactic use of antiemetics and documentation may have confounded our adverse event rates. A potential advantage of fentanyl that our study did not test was its quicker onset. Doing so would have required assessing pain scores at early, fixed intervals after the initiation of therapy, which would have induced the problems of bias and interference with paramedic work flow mentioned above. Our "second field" pain score reported in Figure 1 was reported only for patients who had more than two field pain measurements and was not recorded at a predetermined interval after the first measurement (average of 10.9 minutes). Even considering these limitations, we did not observe a significant divergence in pain control between the opioids at this interim time point that would have suggested a more rapid onset for fentanyl.

\section{Conclusion}

Morphine and fentanyl provided similar degrees of analgesia in this sample of patients treated for pain in the out-of-hospital setting, although this was achieved with a higher narcotic equivalent dose of fentanyl. Both medications had similarly low rates of adverse events that responded to basic paramedic interventions.

\section{Acknowledgments}

The authors thank Chris Koppenhafer, Dana Zive, and Michele Dove for assistance in obtaining the records for this study.

Supported by the Oregon Clinical and Translational Research Institute (OCTRI), grant number UL1 RR024140 from the National Center for Research Resources (NCRR), a component of the National Institutes of Health (NIH), and NIH Roadmap for Medical Research.

\section{References}

1. Rupp T, Delaney KA. Inadequate analgesia in emergency medicine. Ann Emerg Med. 2004; 43:494-503. [PubMed: 15039693]

2. Alonso-Serra HM, Wesley K, National Association of EMS Physicians Standards and Clinical Practices Committee. Prehospital pain management [National Association of EMS Physicians position paper]. Prehosp Emerg Care. 2003; 7:482-8. [PubMed: 14582104]

3. Dailey MW, Burton J, Day L, DeMartino W. Prehospital analgesic protocols in the United States: a national survey [abstract]. Acad Emerg Med. 2009; 16(4 suppl):S197. 
4. Braude D, Richards M. Appeal for fentanyl prehospital use. Prehosp Emerg Care. 2004; 8:441-2. [PubMed: 15626010]

5. Claxton AR, McGuire G, Chung F, Cruise C. Evaluation of morphine versus fentanyl for postoperative analgesia after ambulatory surgical procedures. Anesth Analg. 1997; 84:509-14. [PubMed: 9052292]

6. McLean SA, Domeier RM, DeVore HK, Hill EM, Maio RF, Fred- eriksen SM. The feasibility of pain assessment in the prehospital setting. Prehosp Emerg Care. 2004; 8:155-61. [PubMed: 15060849]

7. MD Consult Drug Monograph, Fentanyl [Internet]. Tampa, FL: Gold Standard; [August 13, 2009]. Available at: http://www.mdconsult.com/das/pharm/body/154020550-6/0/full/245 Updated July 29, 2009

8. Sacchetti A, Senula G, Strickland J, Dubin R. Procedural sedation in the community emergency department: initial results of the ProSCED registry. Acad Emerg Med. 2007; 14:41-6. [PubMed: 16946280]

9. Deitch K, Chudnofsky CR, Dominici P. The utility of supplemental oxygen during emergency department procedural sedation with propofol: a randomized controlled trial. Ann Emerg Med. 2008; 52:1-8. [PubMed: 18294729]

10. Deyo RA, Cherkin DC, Ciol MA. Adapting a clinical co- morbidity index for use with 1CD-9-CM administrative databases. J Clin Epidemiol. 1992; 45:613-9. [PubMed: 1607900]

11. WHO Collaborating Centre for Drug Statistics Methodology. About the ATC/DDD System [Internet]. Oslo, Norway: 2009 [July 8, 2009]. Available at: http://www.whocc.no/atcddd/

12. Bijur PE, Latimer CT, Gallagher EJ. Validation of a verbally administered numerical rating scale of acute pain for use in the emergency department. Acad Emerg Med. 2003; 10:390-2. [PubMed: 12670856]

13. Bijur PE, Chang AK, Gallagher EJ. The minimum clinically significant difference in pain in older ED patients decreases over time [abstract]. Acad Emerg Med. 2009; 16(4 suppl):S33.

14. Galinski M, Dolveck F, Borron S, et al. A randomized, doubleblind study comparing morphine with fentanyl in prehospital analgesia. Am J Emerg Med. 2005; 23:114-9. [PubMed: 15765326]

15. Berthier F, Potel G, Leconte P, Touze MD, Baron D. Comparative study of methods of measuring acute pain intensity in an ED. Am J Emerg Med. 1998; 16:132-6. [PubMed: 9517686]

16. Kanowitz A, Dunn TM, Kanowitz EM, Dunn WW, Vanbuskirk K. Safety and effectiveness of fentanyl administration for prehospital pain management. Prehosp Emerg Care. 2006; 10:1-7. [PubMed: 16418084]

17. Nielsen ND, Dahl MK, Hansen PA. Safety and efficiency of prehospital pain management with fentanyl administration by emergency medical technicians. Abstract at the 4th Mediterranean Emergency Medicine Congress, 2007. J Emerg Med. 2007; 33(3):335.

18. Thomas SH, Rago O, Harrison T, Biddinger PD, Wedel SK. Fen- tanyl trauma analgesia use in air medical scene transports. J Emerg Med. 2005; 29:179-87. [PubMed: 16029830]

19. DeVellis P, Thomas SH, Wedel SK, Stein JP, Vinci RJ. Prehospital fentanyl analgesia in airtransported pediatric trauma patients. Pediatr Emerg Care. 1998; 14:321-3. [PubMed: 9814395]

20. Raghunathan TE, Lepkowski JM, Hoewyk JV, Solenberger P. A multivariate technique for multiply imputing missing values using a sequence of regression models. Surv Methodol. 2001; 27(1):85-95.

21. Newgard CD. The validity of using multiple imputation for missing out-of-hospital data in a state trauma registry. Acad Emerg Med. 2006; 13:314-24. [PubMed: 16495420]

22. Haukoos JS, Newgard CD. Advanced statistics: missing data in clinical research-part 2: multiple imputation. Acad Emerg Med. 2007; 14:669-78. [PubMed: 17595237]

23. Little, RJA.; Rubin, DB. Statistical Analysis with Missing Data. Second. Hoboken, NJ: WileyInterscience; 2002.

24. Silvfast T, Saarnivaara L. Comparison of alfentanil and morphine in the prehospital treatment of patients with acute ischaemic-type chest pain. Eur J Emerg Med. 2001; 8:275-8. [PubMed: 11785593] 
25. Weldon E, Grierson R, Ariano R. Comparison of fentanyl and morphine in the prehospital treatment of patients with is-chemic type chest pain [abstract]. Prehosp Emerg Care. 2008; 12:95101.

26. Thomson Micromedex Healthcare. DRUGDEX Evaluations, Fentanyl [Internet]. Ann Arbor, MI: 2009 [July 8, 2009]. Available at: http://www.thomsonhc.com

27. Red Book, Pharmacy's Fundamental Reference. Montvale, NJ: Thomson Healthcare; 2008. p. 437-590.

28. Haukoos JS, Newgard CD. Advanced statistics: missing data in clinical research—part 1: an introduction and conceptual framework. Acad Emerg Med. 2007; 14:662-8. [PubMed: 17538078] 
9.0

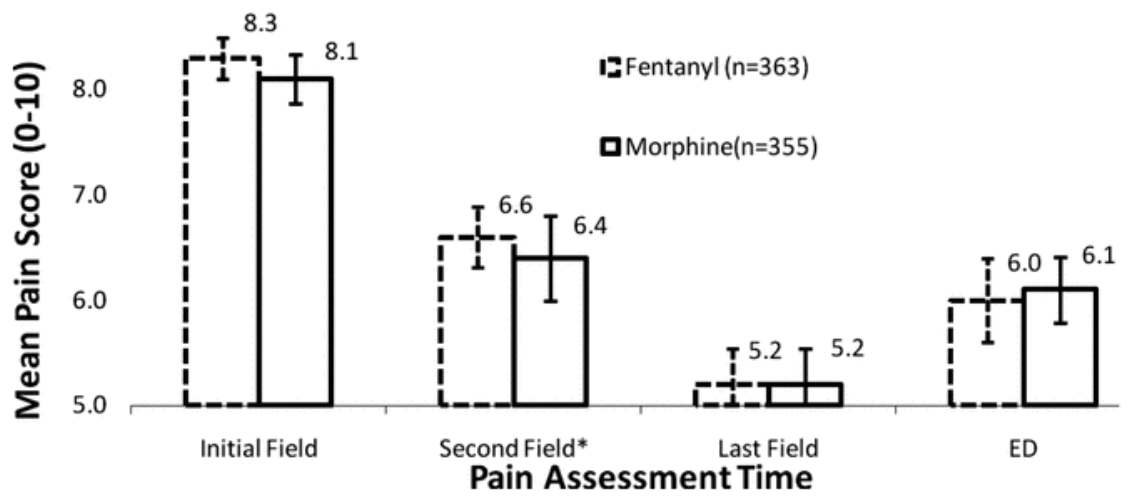

Figure 1.

Pain scores with morphine and fentanyl administration out of hospital and in the emergency department $(\mathrm{ED})(\mathrm{n}=718)$. Error bars show the $95 \%$ confidence interval. *Only for patients with more than two field pain scores documented. 


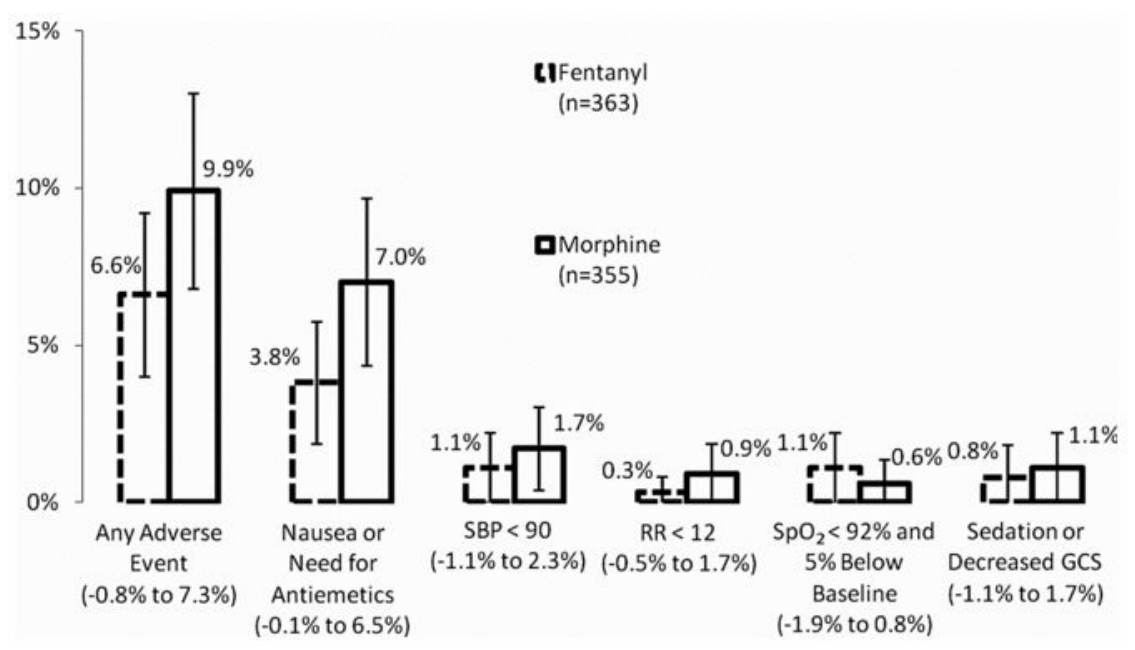

Figure 2.

Adverse events associated with morphine and fentanyl observed during the out-of-hospital period. Error bars show the $95 \%$ confidence intervals. Numbers in parentheses show the 95\% confidence intervals for the difference between the proportions. GCS = Glasgow Coma Scale (score); RR = respiratory rate (in breaths/min); $\mathrm{SBP}=$ systolic blood pressure (in $\mathrm{mmHg}) ; \mathrm{SpO}_{2}=$ oxygen saturation. 


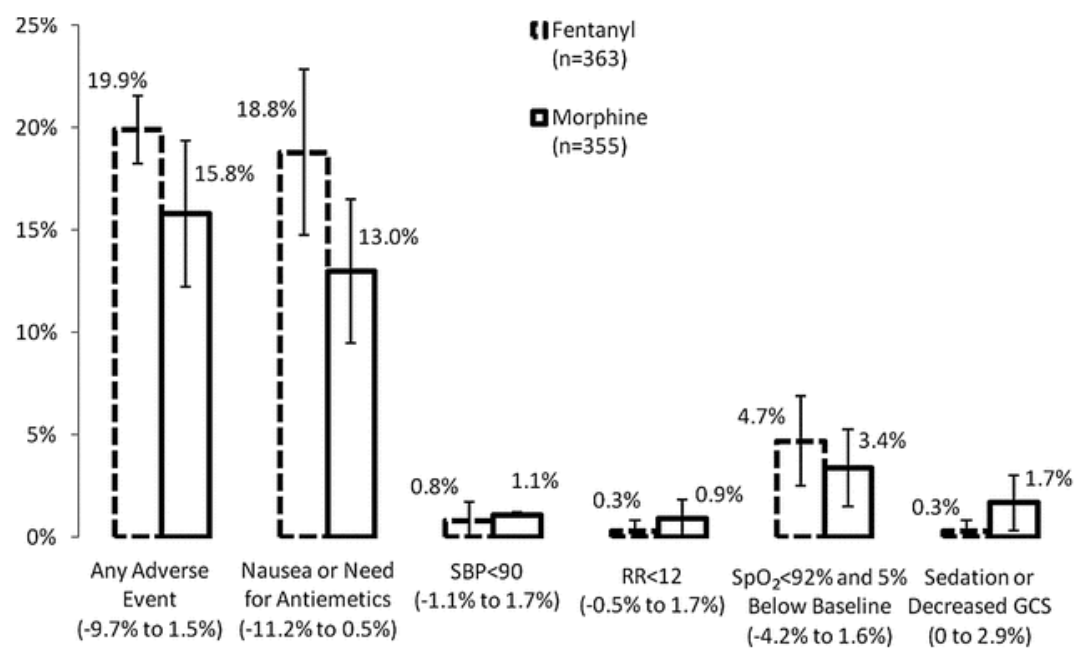

Figure 3.

Adverse events associated with morphine and fentanyl observed in the emergency department. Error bars show the $95 \%$ confidence intervals. Numbers in parentheses show the $95 \%$ confidence intervals for the difference between the proportions. GCS = Glasgow Coma Scale (score); RR = respiratory rate (in breaths/min); $\mathrm{SBP}=$ systolic blood pressure (in $\mathrm{mmHg}$ ); $\mathrm{SpO}_{2}=$ oxygen saturation. 


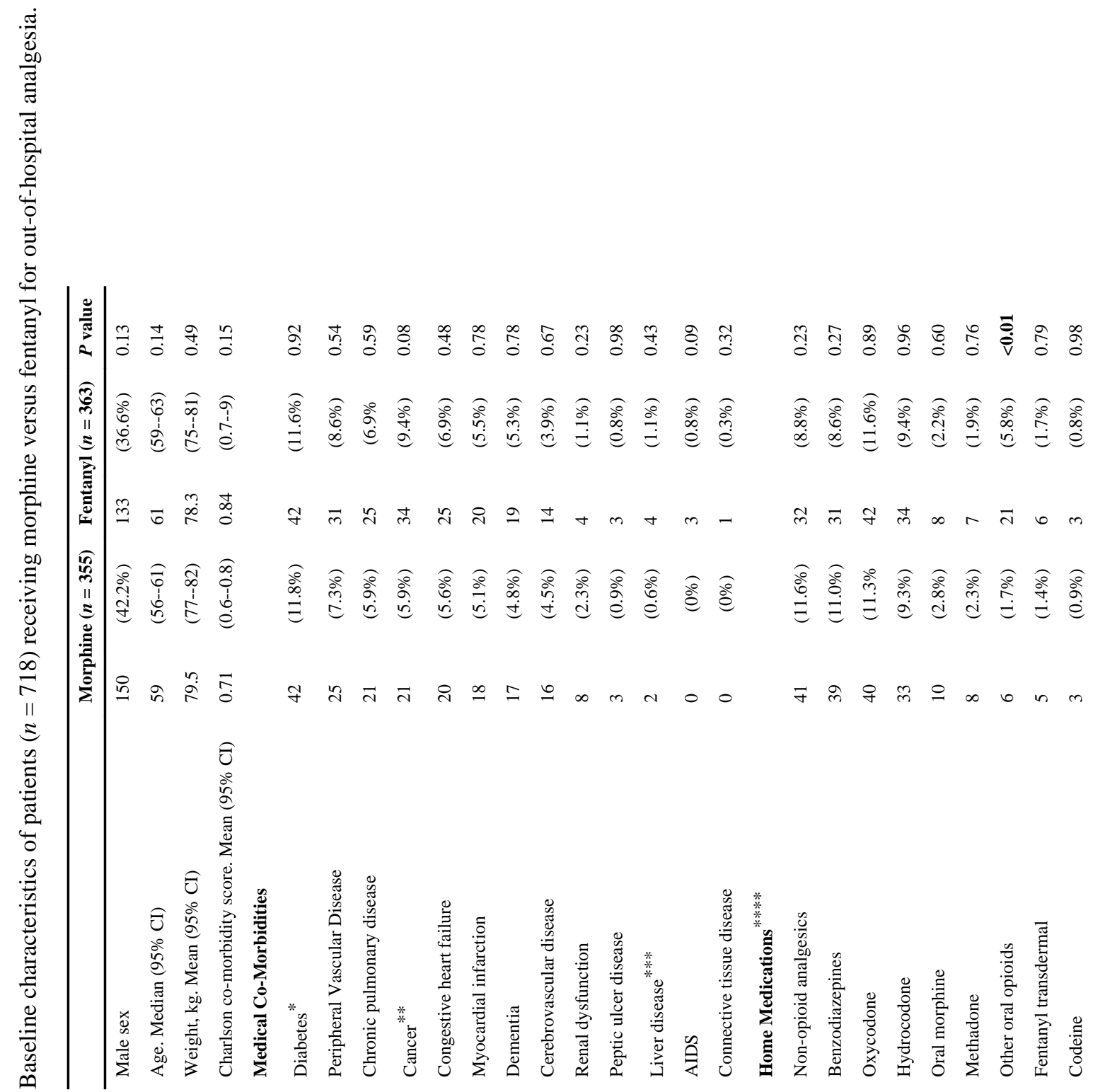




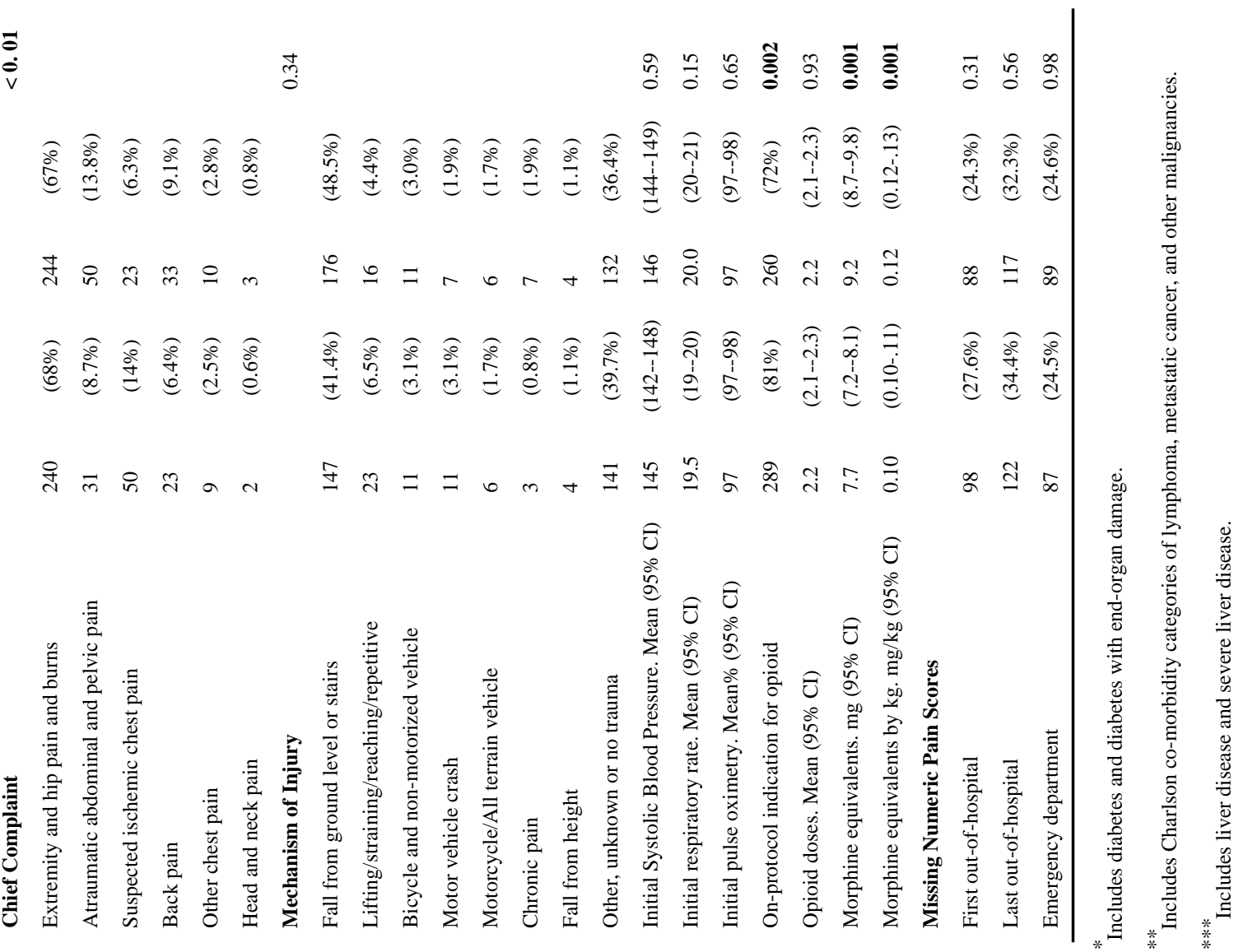


Table 2

Multivariable Linear Model for Change in Pain Score from the First to the Last Field Measurements

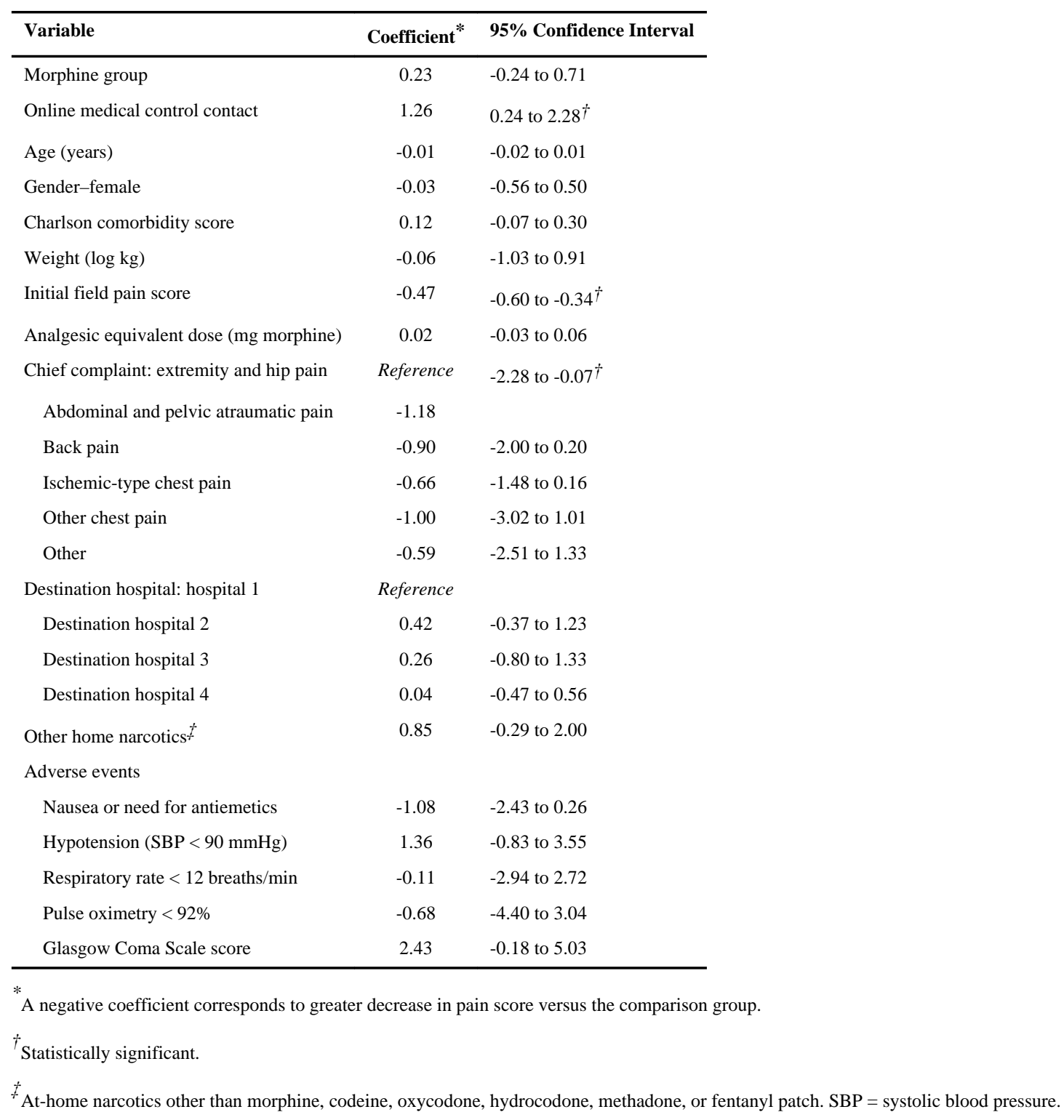

